\title{
Hybridizing Differential Evolution with a Genetic Algorithm for Color Image Segmentation
}

\author{
R. V. V. Krishna \\ Aditya College of Engineering \& Technology \\ Kakinada, India \\ rvvkrishnaece@gmail.com
}

\author{
S. Srinivas Kumar \\ Jawaharlal Nehru Technological University \\ Kakinada, India \\ samay_ssk2@yahoo.com
}

\begin{abstract}
This paper proposes a hybrid of differential evolution and genetic algorithms to solve the color image segmentation problem. Clustering based color image segmentation algorithms segment an image by clustering the features of color and texture, thereby obtaining accurate prototype cluster centers. In the proposed algorithm, the color features are obtained using the homogeneity model. A new texture feature named Power Law Descriptor (PLD) which is a modification of Weber Local Descriptor (WLD) is proposed and further used as a texture feature for clustering. Genetic algorithms are competent in handling binary variables, while differential evolution on the other hand is more efficient in handling real parameters. The obtained texture feature is binary in nature and the color feature is a real value, which suits very well the hybrid cluster center optimization problem in image segmentation. Thus in the proposed algorithm, the optimum texture feature centers are evolved using genetic algorithms, whereas the optimum color feature centers are evolved using differential evolution.
\end{abstract}

Keywords-differential evolution; genetic algorithm; clustering; segmentation; hybrid algorithms; rough sets; fuzzy sets; soft sets

\section{INTRODUCTION}

Color image segmentation is to divide a chromatic image into different homogeneous and connected regions based on color, texture and their combination [10]. It is an essential part of image analysis and decides the final output of any image analysis task. Segmentation methods can be categorized based on the technique used (edge based, region based, fuzzy and neural networks based, feature clustering based etc). In this paper, the color image segmentation performed is based on feature clustering. The consistency of clustering based segmentation methods such as k-means, Fuzzy-c-Means, etc are limited by the initially chosen cluster centers, and also on the cardinality of chosen cluster centers. This problem is solved by using evolutionary computing in this paper. The population of initial cluster centers is formed by the repeated application of the Soft Rough Fuzzy-c-Means (SRFCM) clustering algorithm. The optimal cluster centers are evolved by applying a hybrid of two exemplary evolutionary algorithms, Differential Evolution (DE) and Genetic Algorithms (GAs). Genetic Algorithms are heuristic global search methods that mimic the process of natural selection and uses fixed-length strings to represent possible solutions. GAs are driven by a fitness function defined to evaluate a solution's ability to deal with a given task, ending up in bringing out an optimum solution [17]. DE is a simple yet powerful algorithm for real parameter optimization proposed in [21]. DE is different from traditional algorithms in the sense that it perturbs the current generation population members with the scaled differences [22]. DE has been applied to a number of real world problems due to its simple and robust nature. Similar hybrid approaches have been reported in the literature for a variety of applications.

An Improved differential evolution method has been proposed in to optimize the multi-objective parameters in fuzzy clustering [14] as well as GAs [4]. In [11] a recurrent network design by hybridizing GA and Particle Swarm Optimization (PSO) was proposed. Hybridization of DE and Quantum PSO (QPSO), named DEQPSO, was proposed in [7]. In [23], a hybrid of GA and DE for solving the unit commitment scheduling problem in power systems was proposed. In [25], the multi objective optimization proposed employed GAs, PSO, and Adaptive Metropolis Search (ANS). In general, it can be considered that GAs very ably handle binary variables and $\mathrm{DE}$ is more capable in handling continuous variables. Motivated by this, a composite feature of both color and texture is formed to solve the color image segmentation problem. Texture consists the binary part of the solution and color the real part. GA operates on the texture part and DE operates on the color part, so that the hybrid optimizer effectively explores both the binary and real search domain.

\section{COLOR AND TEXTURE FEATURE EXTRACTION}

\section{A. Color Feature Extraction}

The color image consists of multiple bands, with each band containing a range of intensity values. The color image is represented in different color spaces RGB, Lab, HIS, HSV etc. It is very important to extract the effective pixel-level image features [26]. Here, the Lab color model is used for color feature extraction because it is very convenient to measure small color difference. The pixel level color feature of a color component can be computed as follows: (1) Prepare a window 
of size $3^{\times} 3$ for the construction of pixel-level color feature, (2) Calculate pixel wise color feature related to the color component using pixel homogeneity, extracted from the image, so that it reflects the uniformity of an image object. Pixel variance in terms of standard deviation and discontinuity in terms of edge detection, of color component are calculated. The product of normalized standard deviation and normalized edge discontinuity information is deducted from unity to obtain pixel homogeneity of the objects in the image.

\section{B. Texture Feature Extraction: Power Law Descriptor:}

The Weber's law states that the ratio of incremental threshold to the background intensity is a constant [2]. In short, Weber's law says that the size of a just noticeable difference is a constant proportion of the original stimulus value. In [5], it was proposed that this could be employed as a texture descriptor. But in [1], it was stated that empirical data such as an image does not always fit well into weber's law and a modification was suggested (Guilford power law) that could model the perception of human beings better.

\section{SOFT ROUGH FUZZY C-MEANS ALGORITHM (SRFCM)}

SRFCM has its roots in the k-means algorithm. This basic algorithm was evolved to the Rough k-means (RKM) that was proposed in [12] borrowing some of the concepts of rough set theory [19] and rough fuzzy c-means algorithm which was applied to medical image segmentation problem [15]. In a segmentation problem, the task is to know whether two objects are similar, and a measure of their similarity is needed for clustering different objects in an image [13]

The fundamental steps of SRFCM are as follows: (1) assume $m$ random initial cluster prototypes (2) find membership $u_{i k}$ between $m$ cluster centers and $k$ data points (3) allocate each data point to the lower or upper approximation and (4) make the final assignment based on the difference between the highest and next highest membership of a data point in all clusters (5) compute the similarity of sample points soft set to the cluster centre soft set, calculate the maximum similarity and assign a pixel to a cluster to which it has maximum similarity after fuzzification (6) compute the updated cluster prototype for each cluster (7) iterate and run steps 2-6 until there are no further changes in cluster centroids.

\section{DegaH ALGORITHM}

The proposed algorithm is named DEGAH due to it being a DE and GAs Hybrid. Its basic flowchart is shown in Figure 1.

\section{A. Chromosome Formation \& Population Initialization}

In this image segmentation problem based on clustering, every pixel in the image is represented by a concatenation of color and texture features to form an integrated feature of size $\left[1 * n_{1} n_{2}\right]$ where $n_{1}$ and $n_{2}$ are the lengths of binary texture feature and real color features respectively. The individual chromosome in the DEGAH algorithm is formed by first running the SRFCM clustering algorithm, to obtain $k$ cluster centers, each of size $\left[1 * n_{1} n_{2}\right]$. The resulting, $k$ cluster centers or prototypes are arranged in line to form a chromosome of size $\left[1 * k n_{1} n_{2}\right]$. This process of SRFCM clustering is repeated $N$ times to form an initial population of size $\left[N * k n_{1} n_{2}\right]$.The typical value of $\mathrm{N}=20$ is used in this experimentation.

\section{B. Fitness Evaluation}

In evolutionary algorithms, the right of way of the genetic strings is ordered according to the fitness values calculated based on a cost function. By maximizing or minimizing the fitness values at each generation, the genetic string with the global optimum could be identified to be the terminal clustering result. Currently, many indices, such as K-means index (KMI), separation index (SI), partition separation index (PASI), Davies-Bouldin index (DBI), and fuzzy C-means index (FCMI), are used as the fitness functions of evolutionary algorithms. In all the referred indices, the intra-cluster distance is minimized and inter-cluster distance is maximized. In our experimental work the Davies-Bouldin index (DBI) is chosen as the fitness function owing to its optimal computational complexity [8].

\section{Elitism}

Elitism entails upon copying a small proportion of the fittest solutions, unaltered into the future generation. This ensures that EA's does not waste re-discovering previously discarded partial solutions. Candidate solutions that are preserved unchanged through elitism remain eligible for selection as parents when breeding the remainder of the next generation. In our proposed algorithm, the top (N/5) ranked chromosomes are preserved and named as elite and are directly eligible for crossover in the next generation.

\section{Chromosome Variation}

The hybridization of the GA and DE takes place in the variation step at each generation. In the variation step, the optimum binary texture feature centers are obtained with GA operators and the optimum real color feature centers are obtained using DE operators as discussed below. All the initial population of the combined color and texture centers are evaluated by DBI. If the termination criteria are not satisfied, the texture portion of the solution is speckled by a Genetic Algorithm and the color portion is scrambled by Differential Evolution. The GE variation operation involves two operations viz crossover and mutation. In crossover, two parent chromosomes are mixed, based on two point crossover and crossover probability to yield two offspring chromosomes. The color component is varied by using Differential Evolution. DE also performs the two operations, mutation and crossover.

\section{E. Termination criteria}

Two termination conditions as shown below are employed. The algorithm is terminated if any of the termination conditions is met. Condition 1: If for 200 consecutive generations, the objective function of the best solution (found so far) does not improve by 0.01 and Condition 2: If the maximum allowed generations is reached. 


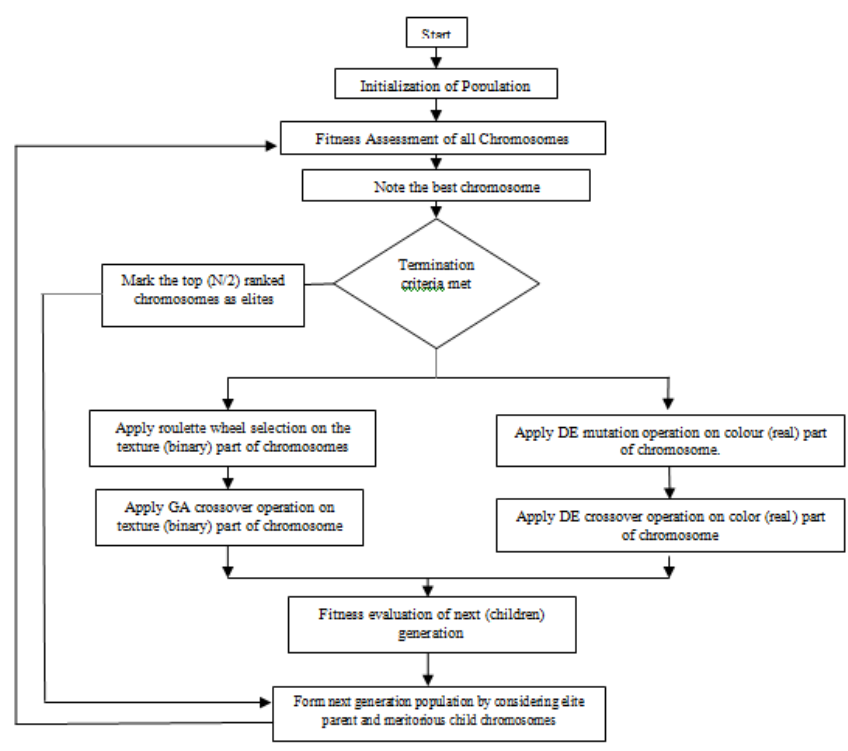

Fig. 1. The DEGAH flowchart

\section{PERFORMANCE MEASURES}

There exists many segmentation evaluation measures [6] such as sensitivity, specificity, Precision, Recall, ROC, F-measure, Local consistency Error, Global consistency Error etc. The performance measures proposed in [16] which are Rand Index (RI), Variation of Information (VOI), Global Consistency Error (GCE), and Boundary Displacement Error (BDE) are used in evaluating and comparing our segmentation results with benchmark algorithms.

\section{A. Rand Index}

The Rand index indicates the proportion of pixels which are in agreement between the Computed Segmentation (CS) and the Ground Truth (GT). [16].

\section{B. Variation of Information}

The variation of information (VOI) is a measure that specifies the variation between computed segmentation and ground Truth. The difference between average conditional entropy of computed segmentation (CS) and Ground Truth (GT) is used to measure the vagueness in $\mathrm{CS}$ which cannot be expressed by GT.

\section{Global Consistency Error}

Global consistency error is a measure of the limits to which the computed segmentation can be seen as transformation of Ground Truth towards Computed Segmentation. Similar segmentations match, as both have genesis in the same image, but undergo segmentation at different scales. If one segment is proper subset of the other, then the pixel lies in an area of refinement, and the error should be zero. If there is no subset relationship, then the two regions overlap.

\section{Boundary Displacement Error}

The Boundary Displacement Error is a measure of the displacement error averaged between boundary pixels in computed segmentation and the nearest boundary pixels in the ground truth. BDE should be low for good segmentation.

\section{RESULTS AND DISCUSSION}

In [8], the well-known J-SEGmentation (JSEG) algorithm, which combines both quantization process and clustering techniques for extraction of color-texture cues in images was proposed. Mean Shift clustering in sync with edge information was employed for edge detection and image segmentation [5]. The proposed algorithm is applied on five natural color images (bear, boat, church, horse, tiger) obtained from Berkeley Segmentation Data Base. Results are compared with the GA without hybridization, and the works in [5] and [8] which are known to be bench mark algorithms in the field of color image segmentation. Results are summarized in Tables I-II and depicted in Figure 2.

TABLE I. RI \& VOI

\begin{tabular}{|c|c|c|c|c|c|c|c|c|}
\hline \multirow{2}{*}{ Image } & \multicolumn{3}{|c|}{ RI } & \multicolumn{4}{c|}{ VOI } \\
\cline { 2 - 9 } & DEGAH & GA & JSEG [8] & EDISION [5] & DEGAH & GA & JSEG [8] & EDISION [5] \\
\hline 1 & 0.69 & 0.39 & 0.61 & 0.68 & 1.81 & 4.52 & 2.09 & 2.55 \\
\hline 2 & 0.62 & 0.57 & 0.45 & 0.46 & 3.01 & 3.60 & 3.64 & 5.61 \\
\hline 3 & 0.73 & 0.72 & 0.45 & 0.67 & 2.52 & 2.66 & 3.03 & 3.06 \\
\hline 4 & 0.65 & 0.62 & 0.45 & 0.46 & 2.98 & 3.33 & 3.34 & 5.33 \\
\hline 5 & 0.82 & 0.71 & 0.47 & 0.54 & 2.53 & 2.62 & 2.63 & 4.15 \\
\hline
\end{tabular}

TABLE II. GCE \& BDE

\begin{tabular}{|c|c|c|c|c|c|c|c|c|}
\hline \multirow{2}{*}{ Image } & \multicolumn{4}{|c|}{ GCE } & \multicolumn{4}{c|}{ BDE } \\
\cline { 2 - 9 } & DEGAH & GA & JSEG [8] & EDISION [5] & DEGAH & GA & JSEG [8] & EDISION [5] \\
\hline Bear & 0.14 & 0.19 & 0.19 & 0.19 & 5.01 & 5.99 & 6.12 & 6.00 \\
\hline Boat & 0.31 & 0.31 & 0.32 & 0.31 & 3.45 & 4.21 & 4.22 & 3.45 \\
\hline Church & 0.17 & 0.19 & 0.21 & 0.19 & 3.85 & 4.95 & 10.24 & 8.74 \\
\hline Horse & 0.24 & 0.24 & 0.25 & 0.24 & 3.01 & 3.06 & 7.29 & 5.86 \\
\hline Tiger & 0.16 & 0.18 & 0.20 & 0.19 & 4.36 & 4.11 & 13.05 & 9.49 \\
\hline
\end{tabular}



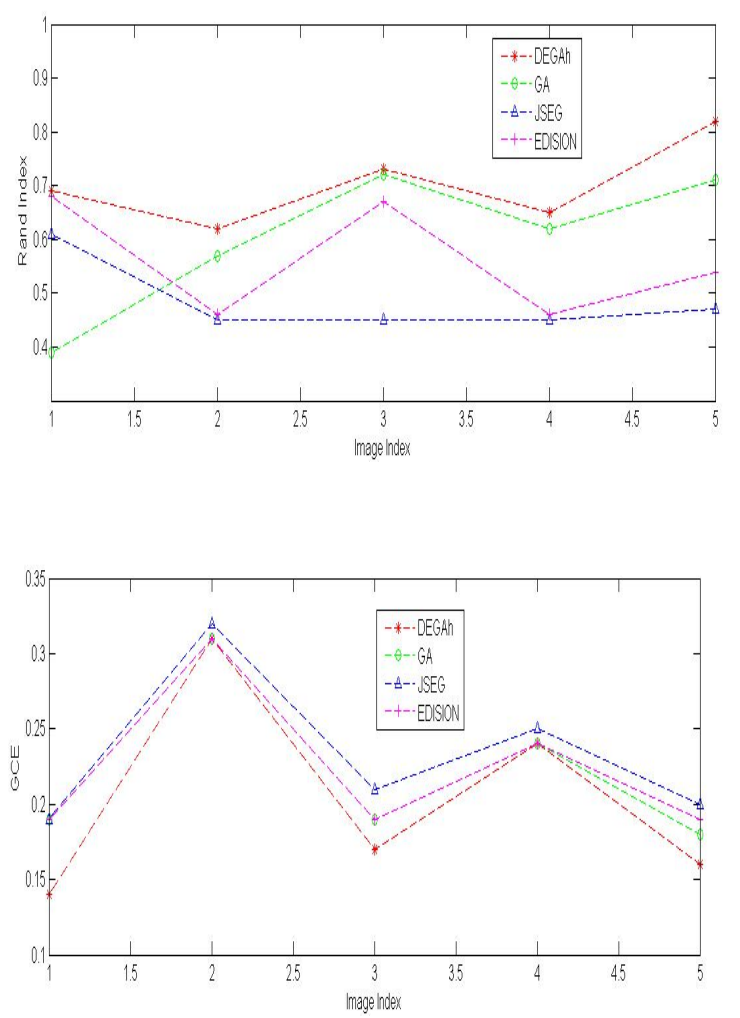

Fig. 2. A sum of the results
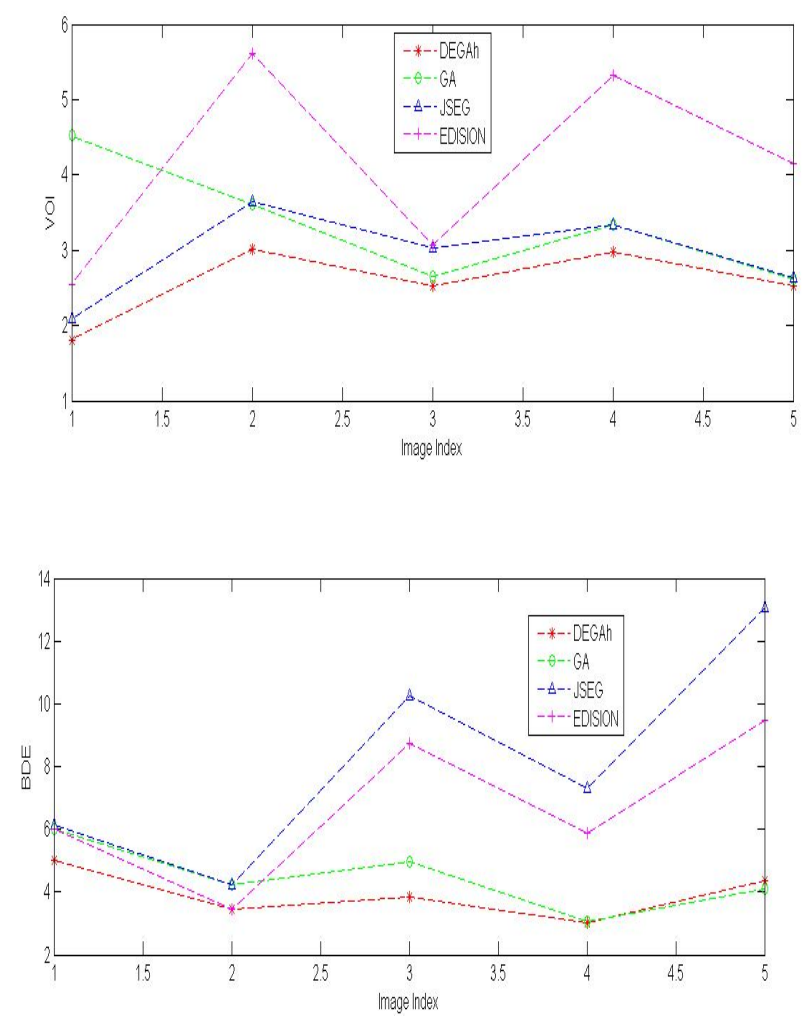

[4] S. Bandyopadhyay, U. Maulik, "Genetic Clustering for Automatic Evolution of Clusters and Application to Image Classification", Pattern Recognition,Vol. 35, pp. 1197-1208, 2002

The approach proposed in this paper is a robust and novel technique which hybridizes two exemplary evolutionary algorithms (Differential Evolution and Genetic Algorithms) for solving the color image segmentation problem. Extensive experimentation was conducted on images from Berkeley segmentation database. The effectiveness of the proposed algorithm is demonstrated through the comparison with other state of the art algorithms. The results shows that, in the proposed algorithm, inter cluster distance has been maximized and intra clustering distance has been minimized. Various performance metrics have been compared and the proposed algorithm shows better results compared with other existing benchmark algorithms. The proposed algorithm can also be extended by hybridizing with other evolutionary algorithms like simulated annealing and PSO, which further increases the clustering accuracy.

\section{REFERENCES}

[1] T. Augustin, "The problem of meaningfulness: Weber's law, Guilford's power law, and the near-miss-to-Weber's law", Mathematical Social Sciences, Vol. 57, No. 1, pp. 117-130, 2009

[2] A. K. Jain, Fundamentals of Digital Signal Processing, Englewood Cliffs, NJ: Prentice-Hall, 1989

[3] S. Bandyopadhyay, U. Maulik, "Nonparametric Genetic Clustering: Comparison of Validity Indices", in IEEE Trans. Systems, Man, and Cybernetics - Part C: Application and Reviews, Vol. 31, pp. 120-125, 2001
[5] J. Chen, S. Shan, G. Zhao, X. Chen, W. Gao, M. Pietikäinen, “A robust descriptor based on Weber's law", IEEE Conference on Computer Vision and Pattern Recognition (CVPR), 2008,

[6] C. M. Christoudias, B. Georgescu, P. Meer, "Synergism in lowlevel vision" $16^{\text {th }}$ IEEE Conference on Pattern Recognition, Vol.4, pp 150-155, New York, 2002

[7] Y. Fu. M. Ding, C. Zhou, H. Hu, "Route planning for unmanned aerial vehicle (UAV) on the sea using hybrid differential evolution and quantum-behaved particle swarm optimization". IEEE Transactions on Systems, Man, and Cybernetics: Systems, Vol. 43, No. 6, pp. 1451-1465, 2013 IEEE Transactions on Pattern Analysis and Machine Intelligence, Vol. 2, pp. 224-227, 1979

[9] Y. Deng, B. S. Manjunath, "Unsupervised Segmentation of color-Texture regions in Images and Video", IEEE Transactions on Pattern Analysis and Machine Intelligence, Vol. 23, No. 8, pp. $800-810,2001$

[10] D. E. Ilea, P. F. Whelan, "Image Segmentation based on the integration of color texture descriptors-A review", Pattern Recognition, Vol. 44, No. 10-11, pp. 2479-2501, 2011

[11] C. Juang, C. Lin, "A recurrent self-organizing neural fuzzy inference network", IEEE Transactions on Neural Networks, Vol. 10, No. 4, pp. 828-845, 1999

[12] P. Lingras, C. West "Interval set clustering of web users with rough k-means", Journal of Intelligent Information Systems, Vol. 23, No. 1, pp. 5-16, 2004

[13] P. Majumdar, S. K. Samanta, "On similarity measures of fuzzy soft sets" International Journal of Advanced Soft Computing Applications, Vol. 3, No. 2, pp. 1-8, 2011
[8] D. L. Davies, D. W. Bouldin. "A cluster separation measure", 
[14] U. Maulik, I. Saha, "Modified Differential Evolution based fuzzy clustering for pixel classification in remote sensing imagery", Pattern Recognition, Vol. 42, No. 9, pp. 2135-2149, 2009

[15] S. Mitra. H. Banka, W. Pedrycz, "Rough Fuzzy Collaborative clustering", IEEE Transactions on Systems, Man, and Cybernetics, Vol. 36, No. 4, pp. 795-805, 2006

[16] A. Mukhopadhyay, U. Maulik, S. Bandyopadhyay, "Multiobiective genetic algorithm-based fuzzy clustering of categorical attributes". IEEE Transactions on Evolutionary Computation, Vol.13, No. 5, pp. 991-1005, 2009

[17] A. Munawar, M. Wahib, M. Munetomo, K. Akama, "A survey: Genetic algorithms and the fast evolving world of parallel computing", 10th IEEE International Conference on High Performance Computing and Communications (HPCC '08), pp. 897-902. September 25-27, 2008

[18] F. Neri, V. Tirronen, "Recent advances in differential evolution: a survey and experimental analysis", Artif. Intell. Rev., Vol. 33, pp. 61-106, 2010

[19] Z. Pawalak. Rough sets, Theoretical aspects of Reasoning about data, Dordrecht, The Netherlands: Kluwer, 1991

[20] P. Maji, S. K. Pal, "A hybrid clustering algorithm using rough and fuzzy sets", Fundamental Informaticae, Vol. 80, pp. 475496, 2007

[21] R. Storn, K. V. Price, Differential evolution: a simple and efficient adaptive scheme for global optimization over continuous spaces, International Computer Science Institute, University of Berkley, CA, USA, Technical Report, TR- 95012,1995

[22] S. Das, P. N. Suganthan, "Differential evolution: a survey of the state-of-the-art", IEEE Trans. Evol. Comput. Vol. 15, No. 1, pp. 4-31, 2011

[23] A. Trivedi, D. Srinivasan, S. Biswas, T. Reind, "Hybridizing genetic algorithm with differential evolution for solving the unit commitment scheduling problem", Swarm and Evolutionary Computation, Vol. 23, pp. 50-64, 2015

[24] U. Maulik, S. Bandyopadhyay, "Genetic algorithm-based clustering technique", Pattern Recognition, Vol. 33, No. 9, pp. 1455-1465, 2000

[25] J. Vrugt, B. Robinson, "Improved evolutionary optimization from genetically adaptive multi method search", Proceedings of the National Academy of Sciences, Vol. 104, No. 3, pp. 708711,2007

[26] X. Y. Wang, Wang. T, Juan B“"Color Image Segmentation using pixel wise support vector machine cklassification", Pattern Recognition, Vol. 44, No. 4, pp. 777-787, 2011 